\title{
Emociones positivas del alumnado de Educación Secundaria en las prácticas de baloncesto en Educación Física Positive emotions of the students of Secondary Education in the practices of basketball in Physical Education \\ *Felipe Nicolás Mujica Johnson, *Ana Concepción Jiménez Sánchez *Universidad Politécnica de Madrid (España)
}

\begin{abstract}
Resumen: La investigación de las emociones en Educación Física se debe a la necesidad de comprender los diferentes significados que dichos estados psicológicos pueden representar. El objetivo de esta investigación fue interpretar la percepción y atribución del alumnado sobre las emociones positivas para el bienestar subjetivo durante la práctica de baloncesto en Educación Física, en función del tipo de tarea motriz, el género y el centro educativo. Para aproximarse al fenómeno emocional, se ha decidido utilizar una metodología cualitativa. Participaron 20 alumnas y 24 alumnos pertenecientes a dos centros educativos de la Comunidad de Madrid. Los datos fueron recogidos por medio de entrevistas semi-estructuradas y diarios personales, los cuales se sometieron a un análisis de contenido deductivo-inductivo con ayuda del programa informático Atlas.ti 7.5. Los resultados muestran 18 códigos o atribuciones emocionales que se refieren a la alegría, el entusiasmo, la seguridad y la diversión. Según el tipo de tarea motriz, el género y el centro educativo se identificaron diferencias y similitudes, destacando el rol de la competencia y la relación con una cultura deportiva de baloncesto en cada centro educativo. Se concluye que, para promover el bienestar subjetivo del alumnado en las prácticas de baloncesto en Educación Física, podría ser adecuado aplicar modelos de enseñanza alternativos del deporte, estrategias adaptativas y un clima de logro orientado a la tarea.
\end{abstract}

Palabras clave: afectividad, baloncesto, educación física, género, cultura deportiva.

\begin{abstract}
Investigation of emotions in Physical Education is due to the need to understand the different meanings that these psychological states can represent. The objective of this research was to interpret the perception and attribution of the students about the positive emotions for the subjective well-being during the practice of basketball in Physical Education, depending on the type of motor task, gender and educational center. To approach the emotional phenomenon, it has been decided to use a qualitative methodology. 20 girls and 24 boys from two educational centers in the Community of Madrid participated. The data was collected through semi-structured interviews and personal diaries, which have been subjected to a deductive-inductive content analysis with the help of the Atlas.ti 7.5 software. The results indicated 18 emotional codes or attributions found for joy, enthusiasm, security and fun. According to the type of motor task, gender and educational center, differences and similarities are identified, highlighting the role of competition and the relationship with a basketball sports culture in each educational center. It is concluded that, to promote the subjective well-being of the students in the practices of basketball in Physical Education, it could be appropriate to apply alternative teaching models of sport, adaptive strategies and a climate of achievement oriented to the task.
\end{abstract}

Key Words: affectivity, basketball, physical education, gender, sports culture.

\section{Introducción}

En España, diferentes autores han divulgado y, por supuesto, fundamentado la importancia de que la asignatura de Educación Física (EF) aporte a la formación integral de las personas (Cagigal, 1979; Castejón, 2010; Duran et al., 2015; Mujica, 2020). Es decir, que se preocupe de educar las diferentes dimensiones que forman la totalidad de lo humano, por ejemplo, las emociones del alumnado durante su aprendizaje motor (Alcaraz-Muñoz, Alonso \& Yuste, 2017; Alonso, Lagardera, Lavega \& Etxebeste, 2018; Baños et al., 2019; Bermúdez \& SáenzLópez, 2019; Gil-Madrona, Pascual-Francés, Jordá-Espi, Mujica-Johnson \& Fernández-Revelles, 2020; SalgadoLópez, 2014). La legislación del currículo educativo español promueve que, en Educación Secundaria Obligatoria (ESO) y Bachillerato, el alumnado pueda comprender los aspectos emotivos que experimenta durante sus sesiones de EF (Ministerio de Educación, Cultura y Deporte, 2015). En este sentido, el profesorado de EF debería poder facilitar dicha comprensión emocional. Pero, es preciso mencionar que la tarea de comprender las emociones puede ser bastante compleja, ya que podrían estar asociadas a

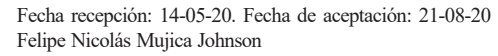

Fecha recepción: 14-05-20. Fecha de aceptación: 21-08-20 fmujica@live.cl múltiples factores subjetivos, intersubjetivos y transubjetivos (Calderón, 2014; Mujica, Orellana \& Concha, 2018). Precisamente, un significado de emoción que representa dicha complejidad es el siguiente:

La emoción no es una simple reacción fisiológica, sino que, a pesar de tener una dimensión de este tipo, también está relacionada con rasgos de personalidad (dimensión psicológica), subculturas específicas (modalidades deportivas -en nuestro caso-, género, edad...) y contextos socioculturales e históricos (Puig, 2012, p. 106).

Para contribuir al entendimiento de las emociones del alumnado en EF, se han desarrollado, en diferentes países, estudios con una metodología de investigación cualitativa. De esta forma, se han podido desvelar algunos significados que, desde la perspectiva estudiantil, reflejan precauciones didácticas que se podrían tomar en consideración en las programaciones curriculares de la asignatura. Sobre todo, para fomentar en el bienestar subjetivo (BS), el aprendizaje motor, la autoestima, la participación en las sesiones de EF, la igualdad de género o el desarrollo moral del alumnado (Alcaraz-Muñoz et al., 2017; Amado, García, Marreiros, López \& del Villar, 2015; Barker, Nyberg \& Larsson, 2019; Leisterer \& Jekauc, 2019; Monforte \& Pérez-Samaniego, 2017; Mujica, Orellana, Aránguiz \& González, 2016). En concreto, algunas investigaciones han reconocido que, desde la perspectiva de 
género, las chicas pierden el entusiasmo y sienten vergüenza ante un discurso de género que menosprecia al género femenino o se burla de ellas en las actividades motrices. Esto se ha identificado en el desarrollo de diferentes contenidos didácticos, entre los que destaca natación, condición física y baloncesto (Azzarito, Solmon \& Harrisson, 2006; Camacho-Miñano \& Aragón, 2014; Mitchell, Gray \& Inchley, 2015; Mujica et al., 2016; Timken, McNamee \& Coste, 2019).

Por otra parte, una investigación cualitativa realizada en un centro educativo de Alemania, que tuvo la participación de 6 chicos y 6 chicas con una edad promedio de 15.6 años, logró identificar motivos que, desde la perspectiva del alumnado, suscitarían emociones positivas para el BS en las sesiones de EF. En concreto, sería el atractivo o agrado que se tiene hacia las actividades de $\mathrm{EF}$, la pertenencia social al grupo curso, la buena competencia motriz percibida y la autonomía que se tendría para aportar aspectos innovadores a las sesiones de EF (Leisterer \& Jekauc, 2019). Dichos resultados, coinciden, en parte, con los de otras dos investigaciones que abordan el excesivo deseo que tuvo el alumnado en algunos centros educativos de Estados Unidos por la práctica de baloncesto en EF (Azzarito et al., 2006; McCaughtry, Barnard, Martin, Shen \& Hodges, 2006). Así, los estudios mencionados explican que, dada la importancia que tiene el baloncesto en la cultura de los adolescentes de dicho país, el alumnado, principalmente hombre, presenta un intenso entusiasmo por jugarlo y competir con sus pares en torno a dicho deporte. Lo cual, en algunas ocasiones, el profesorado lo utilizó como estímulo para conseguir algunas acciones de parte de ellos y, en otras ocasiones, les perjudicó el desarrollo de otros contenidos didácticos, ya que solo estaban dispuestos a practicar baloncesto. En este sentido, dichos estudios expresan el aspecto transubjetivo o cultural de las emociones en EF.

Otro estudio con enfoque cualitativo que indagó ampliamente la dimensión emocional del alumnado en EF es el de Niven, Henretty \& Fawkner (2014). En dicha investigación participaron 38 chicas adolescentes con edades comprendidas entre los 13 y 16 años, las cuales eran parte de dos centros educativos de Escocia. Así, por medio de ocho grupos focales, logró revelar que algunas chicas perciben emociones positivas para el $\mathrm{BS}$ ante la práctica de deportes colectivos y actividades motrices individuales, destacando el baloncesto, el hockey, condición física y danza. A su vez, otras presentan emociones negativas para el BS frente a las mismas actividades deportivas, aludiendo, en algunas ocasiones, a la falta de experiencias motrices en la temática. Por otro lado, algunas pocas chicas se interesan por el fútbol, pero muchas declinaban participar en ese deporte por la presencia de los chicos que hacen muy competitiva la práctica. Además, algunas alumnas plantearon que, para prevenir el miedo y el aburrimiento en EF, sería conveniente que existiese una suficiente variedad de actividades. Sumado a lo anterior, las chicas propusieron más autonomía, con la finalidad de que el alumnado pueda tomar decisiones (Niven et al., 2014). Del mismo modo, otras investigaciones en EF también han presentado como hallazgo un nexo entre emo- ciones positivas para el BS y autonomía del alumnado (García-González, Aibar, Sevil, Almolda \& Julián, 2015; Leisterer \& Jekauc, 2019; Moreno, Hernández \& GonzálezCutre, 2009; Mujica \& Jiménez, 2020; Timken et al., 2019).

El objetivo del presente estudio fue interpretar la percepción y atribución del alumnado sobre las emociones positivas para el BS durante la práctica de baloncesto en $\mathrm{EF}$, en función del tipo de tarea motriz, el género y el centro educativo.

\section{Método}

El presente estudio, enmarcado en un proyecto científico más amplio, se ha desarrollado con una metodología de investigación cualitativa (Ortín, Martín, Mena, Izquierdo \& López, 2018) y, en concreto, con un diseño interpretativo y fenomenológico (Angulo, 2017; Denzin \& Lincoln, 2012; Flick, 2015). Así, se realizó una aproximación a la dimensión emocional del alumnado en su contexto natural o común de EF, en otras palabras, desde una mirada interior del cotidiano proceso pedagógico (Navarro, Jiménez, Rappoport \& Thoilliez, 2017). Además, la interpretación de dicha realidad estuvo dirigida, entre diferentes factores, a la cultura deportiva de los centros educativos incluidos en la investigación.

\section{Participantes}

En la investigación participaron 20 alumnas y 24 alumnos, con un promedio etario de 14, 4 años $(\mathrm{DT}=0.690)$. Al momento del estudio, el alumnado estaba cursando sus estudios en dos centros educativos de la Comunidad de Madrid. En concreto, 11 chicos y 9 chicas eran parte del primer instituto de ESO (IESO1). Por otro lado, 13 chicos y 11 chicas pertenecían al segundo instituto de ESO (IESO2). Por cierto, en cuanto a la cultura deportiva de los centros educativos, solo el IESO2 se identifica ampliamente con el baloncesto. Así, para este estudio se decidió incluir a dichos centros educativos por su relación con una cultura formal de baloncesto. Con respecto a la selección del alumnado participante, se hizo por medio de un muestreo intencional (Navarro et al., 2017) e incluye a estudiantes de tres grupos del curso $3^{\circ}$ de ESO por cada centro educativo. En concreto, se utilizaron dos criterios de inclusión. En primer lugar, se decidió incluir cuatro alumnos y cuatro alumnas por cada grupo que no tuviesen experiencia deportiva en un club deportivo de baloncesto. Y, en tercer lugar, en el caso de que no existieran los suficientes voluntarios con los criterios mencionados, se decidió incluir a estudiantes que tuviesen experiencia en un club deportivo de baloncesto.

La participación en el estudio fue de carácter voluntaria, de modo que el alumnado firmó un consentimiento informado que expresó su deseo de incluirse en la investigación. Además, tuvo conocimiento del anonimato de su identidad y de su derecho a excluirse libremente del estudio. Por último, padre, madre y/o tutor(a), autorizaron la inclusión de cada estudiante en la investigación. Con base en estas consideraciones, un alumno del IESO1 no pudo participar por no ser autorizado por sus representantes legales. Igualmente, una alumna se retiró en la mi- 
tad del estudio, señalando que no deseaba continuar comprometida con el proyecto de investigación.

\section{Instrumentos}

Los datos fueron recogidos a través de entrevistas semi-estructuradas, en concreto, al finalizar una tarea motriz de baloncesto. Cabe destacar que, durante cada sesión de la Unidad Didáctica de baloncesto (UDB), se entrevistó una vez a todo el alumnado participante del estudio que estuvo presente. Dichas entrevistas, dado que se realizaban durante la sesión de EF, fueron diseñadas para demandar brevemente la atención del alumnado. Las entrevistas se enfocaron en cuatro emociones positivas para el BS, precisamente en la alegría, la diversión, la seguridad y el entusiasmo. Dichas emociones fueron seleccionadas por ser reconocidas como emociones positivas para el BS, por haber sido incluidas en otros estudios realizados en EF y por ser de interés para los investigadores (Bisquerra et al., 2016; Leisterer \& Jekauc, 2019; Mujica et al., 2016; Niven et al., 2014; Timken et al., 2019). Previamente el alumnado ya había sido sometido a una toma de conciencia sobre el significado de dichas emociones. Con la finalidad de facilitar el reconocimiento de dichas emociones, durante las entrevistas se le otorgó al alumnado una hoja que las mencionaba. Sin embargo, se les señaló que, también, podían hacer alusión a otra emoción positiva para el BS diferente a la del listado, o sea, diferente a las emociones mencionadas anteriormente. Las entrevistas estuvieron orientadas solo por las siguientes preguntas: a) ¿Qué emociones habéis sentido durante la tarea motriz de baloncesto que acabas de realizar?; b) ¿En qué momento de la tarea habéis sentido dicha(s) emoción(es)?; y c) ¿Por qué piensas que habéis sentido esa(s) emoción(es)? El principal criterio para el diseño de las preguntas, fue que dieran respuesta al objetivo del estudio, es decir, que hicieran referencia a la percepción y atribución emocional.

Por otro lado, se utilizó un diario personal, el cual fue aplicado al término de las sesiones de la UDB. Dicho instrumento incluía las mismas cuatro emociones positivas para el BS mencionadas anteriormente. De esta forma, el alumnado, en el caso de haber percibido una o más de ellas, podía marcarlas y, por ello, debía justificar el momento en que la(s) sintió y a qué motivo la(s) atribuyó. Finalmente, al alumnado se le pidió aportar una estrategia pedagógica que pudiese concretarse en la UDB para fomentar las emociones percibidas. Adicionalmente, el contenido de la entrevista semi-estructurada y del diario personal se validó a través el juicio de cuatro expertos, todos doctores especialistas en el ámbito del deporte y/o la EF. En concreto, los cuatro expertos son académicos de universidades españolas con un promedio de 31.5 años de experiencia docente. Entre los temas que han investigado los expertos se encuentra la iniciación deportiva, baloncesto, didáctica de la EF, ética en el deporte y evaluación de programas de actividad física-deportiva.

\section{Procedimiento}

En el trabajo de campo, en primer lugar, se solicitó la autorización del proyecto de investigación a la dirección de los centros educativos y al departamento de EF. Posteriormente, una vez aprobado el proyecto, se seleccionaron los participantes del estudio y se les informo, específicamente, el proceso de recolección de datos durante la UDB. A su vez, el alumnado firmó un consentimiento de participación voluntario y se les dio la autorización para que sea firmada por sus representantes legales.

De este modo, durante el desarrollo de la UDB, se recogieron datos durante 38 sesiones de EF. Por tanto, se aplicaron 211 entrevistas y 132 diarios personales en ambos centros educativos. A su vez, se fueron transcribiendo y analizando los datos que aportaban cada participante. Finalmente, se realizó una validez comunicativa de la información, de modo que cada estudiante leyó sus datos y confirmó que representaban su percepción emocional.

\section{Análisis de datos}

La transcripción de la información aportada por los participantes dio origen a los documentos primarios, los cuales fueron sometidos a un análisis de contenido que siguió la vía deductiva-inductiva, debido a que «las categorías o familias de códigos del estudio fueron elaboradas a partir del marco teórico, mientras que las sub-categorías o códigos de datos surgieron como resultado del proceso de codificación e interpretación de los datos») (Mujica-Johnson \& Jiménez, 2019). En concreto, las familias de códigos establecidas a priori son las siguientes: a) emociones positivas del alumnado en las tareas motrices con oposición (EPATMCO); b) emociones positivas del alumnado en las tareas motrices sin oposición (EPATMSO); c) emociones positivas del alumnado de género femenino (EPAGF); d) emociones positivas del alumnado de género masculino (EPAGM); e) emociones positivas del alumnado del instituto de educación secundaria 1 (EPAIESO1); f) emociones positivas del alumnado del instituto de educación secundaria 2 (EPAIESO2).

Además, el análisis pasó por una fase de codificación, descripción e interpretación de los datos (Mejía, 2011). Para dicho proceso analítico, se utilizó el software para el tratamiento de información cualitativa Atlas.ti en su versión 7.5. Precisamente, dicho programa informático favoreció la recuperación, asociación y representación conceptual de los datos (González-Campos, Rodríguez-López \& Castañeda-Vásquez, 2018; Pérez-López, MoralesSánchez, Anguera \& Hernández-Mendo, 2015).

En cuanto al rigor metodológico, los datos fueron sometidos, previamente al análisis, a una validez comunicativa por medio de un feedback de los participantes (Hayashi, Abib \& Hoppen, 2019). Y, durante el proceso de análisis de contenido, a una triangulación de datos a nivel personal y temporal (Fusch, Fusch \& Ness, 2018). En efecto, se ha decidido excluir de los resultados los códigos que no alcanzaron una frecuencia mínima de tres personas o tiempos que los fundamentaran. Cabe mencionar, que la frecuencia personal hace referencia a la cantidad de personas que aportaron información sobre cada código. Por otro lado, la frecuencia temporal hace referencia a la cantidad de veces o momentos que se hizo alusión a cada código. 


\section{Resultados}

Las seis familias de códigos que representan las emociones positivas del alumnado durante la UDB se encuentran integradas por un total de 18 códigos, los cuales se presentan en la Tabla 1. Además, dichos códigos incluyen las emociones que, desde la perspectiva del alumnado, han suscitado. Igualmente, se ha agregado la frecuencia personal y temporal de las menciones a cada código.

Tabla 1

Códigos de las emociones positivas del alumnado durante la UDB

\begin{tabular}{lccc}
\multicolumn{1}{c}{ Código } & Emoción & FP & FT \\
\hline Agrado de practicar baloncesto & E, SE, D y A & 39 & 125 \\
Práctica de baloncesto junto a compañeros y amistades & E, SE, D y A & 34 & 75 \\
Positiva auto-percepción de la práctica de baloncesto & E, SE, D, SA y A & 22 & 38 \\
Convicción de poder lograr ejecutar la tarea de baloncesto & E, SE, D y A & 16 & 34 \\
Aprendizaje personal de baloncesto & D, A y E & 11 & 14 \\
Experiencia previa en baloncesto & E, SE, D y A & 8 & 17 \\
Percepción positiva del desempeño colectivo en la práctica de & E, A y SE & 9 & 9 \\
baloncesto & & & \\
Bajo nivel de práctica de baloncesto entre el alumnado & E, SE, D y A & 6 & 7 \\
Desafio motor en las prácticas baloncesto & D, A y E & 6 & 6 \\
Trabajo en equipo & D, A y E & 5 & 7 \\
Competencia motriz & D, A y E & 5 & 6 \\
Apoyo entre el alumnado en la práctica de baloncesto & A y D & 5 & 5 \\
Contribuir al aprendizaje del alumnado & E, SE, D y A & 4 & 5 \\
Triunfo en partidos de baloncesto & D, A y E & 4 & 4 \\
Calidad del ejercicio técnico de baloncesto & D, SE y E & 3 & 3 \\
Práctica autónoma de baloncesto & D, A y E & 3 & 3 \\
Nivel superior de práctica de baloncesto & E y SE & 2 & 6 \\
Alto nivel de práctica de baloncesto entre el alumnado & D, A y E & 2 & 4 \\
\hline FP: Frecuencia personal. FT: Frecuencia temporal. E: Entusiasmo. D: Diversión. A: Alegría. SE: & &
\end{tabular}

Seguridad. SA: Satisfacción. Fuente: Elaboración propi

Con la finalidad de aportar al entendimiento de cada código, a continuación, se menciona una unidad de registro representativa de la percepción emocional del alumnado. El primer código agrado de practicar baloncesto, ha sido descrito de la siguiente forma: «pues, he sentido alegría y diversión, porque como me gusta el baloncesto, me divierte» (E1, IESO2). El segundo código práctica de baloncesto junto a compañeros y amistades, está representado así: «diversión y alegría, porque me siento cómo jugando a este deporte y estoy con mis amigos. El ejercicio también me gustó bastante, pues, porque estoy haciendo contacto con mis compañeros, con el balón y dando seguridad entre nosotros» (E23, IESO2). El tercer código se ha fundamentado de esta forma: «alegría, porque me han salido bien los ejercicios» (E19, IESO1). El cuarto código convicción de poder lograr ejecutar la tarea de baloncesto fue expresado así: «con seguridad, porque sabía que lo iba a hacer bien y tampoco se me da mal esto» (E15, IESO1). El quinto código aprendizaje personal de baloncesto fue argumentado así: «pues, alegría y diversión, porque estábamos aprendiendo a hacer la finta (E12, IESO1)». El sexto código experiencia previa en baloncesto se aprecia en la siguiente cita: «Pues me ha producido seguridad y entusiasmo, porque es algo que ya he hecho y se de sobra, entonces sé que no lo voy a hacer mal» (E20, IESO2). El séptimo código percepción positiva del desempeño colectivo en la práctica de baloncesto, se aprecia en la siguiente frase: «sentí eentusiasmo cuando íbamos a marcar a la canasta y la pelota entraba» (E17, IESO1).

El octavo código, bajo nivel de práctica de baloncesto entre el alumnado está representado por la siguiente idea: «las emociones que he sentido depende del compañero, pero en general todas las positivas, porque muchos de los compañeros no saben y eso me crea mucha seguridad» (E13, IESO2). El noveno código desafío motor en las prácticas baloncesto se ha expresado así: «he sentido diversión porque al ser 1 vs 1 es un ejercicio desafiante» (E1, IESO2). El décimo código trabajo en equipo ha sido mencionado de esta forma: «he sentido entusiasmo porque aunque en otras clases hagamos juegos por equipos, hoy hemos tenido que poner cada uno un poco de nuestra parte, tanto para hacer las defensas como para saber a quién vamos a defender cada uno» (E14, IESO1). El undécimo código competencia motriz, se refleja en esta cita: «diversión y alegría, por estar jugando con un coleguita. Pues, algo de entusiasmo también, por la competición sabes» (E14, IESO2). El duodécimo código apoyo entre el alumnado en la práctica de baloncesto está representado por la siguiente frase: «es bastante divertido tirar y hacer esas cosas, porque es en pareja y pudimos estar ahí animándonos» (E19, IESO2). El decimotercer código contribuir al aprendizaje del alumnado se ha expresado así: «diversión y entusiasmo, por enseñar a otras personas que se me da bien a mí» (E17, IESO2). El decimocuarto código triunfo en partidos de baloncesto se ha mencionado de esta forma: "sentí alegría, seguridad y diversión, porque en el partido que hicimos al final de la clase sabía que íbamos a ganar» (12, IESO1). El decimoquinto código calidad del ejercicio técnico de baloncesto se ha expresado de este modo: «entusiasmo, cuando he realizado la tarea, porque ha sido dinámica y me ha gustado» (E18, IESO1). El decimosexto código práctica autónoma de baloncesto está representado por la siguiente percepción: «me he divertido, porque puedes hacer lo que tú quisieras, haber dentro de unos límites, pero más o menos, luego hoy si me he podido poner con mis amigos y eso está bastante bien» (E7, IESO2). El decimoséptimo código nivel superior de práctica de baloncesto está manifestado así: «las emociones que he sentido depende del compañero, pero en general todas las positivas, porque muchos de los compañeros no saben y eso me crea mucha seguridad» (E13, IESO2). Por último, el decimoctavo código alto nivel de práctica de baloncesto entre el alumnado refleja su percepción por medio de la siguiente percepción: «pues, diversión, porque he jugado con un compañero que sabe jugar» (E7, IESO2).

\section{Emociones positivas según las tareas motrices}

Las familias de códigos EPATMCO y EPATMSO presentaron 10 códigos en común. Es decir, que dichos códigos fueron mencionados por el alumnado en ambos tipos de tareas motrices. En concreto, son los siguientes códigos: a) agrado de practicar baloncesto; b) práctica de baloncesto junto a compañeros y amistades; c) positiva auto-percepción de la práctica de baloncesto; d) convicción de poder lograr ejecutar la tarea de baloncesto; e) aprendizaje personal de baloncesto; f) experiencia previa en baloncesto; g) percepción positiva del desempeño colectivo en la práctica de baloncesto; h) desafío motor en las prácticas baloncesto; g) apoyo entre el alumnado en la práctica de baloncesto; i) nivel superior de práctica de baloncesto.

Por otro lado, se identificaron en las familias de códigos EPATMCO y EPATMSO un total de seis códigos independientes. O sea, que el alumnado solo atribuyó a un 
tipo de tarea motriz. Específicamente, dichas relaciones se exponen en la Figura 1.

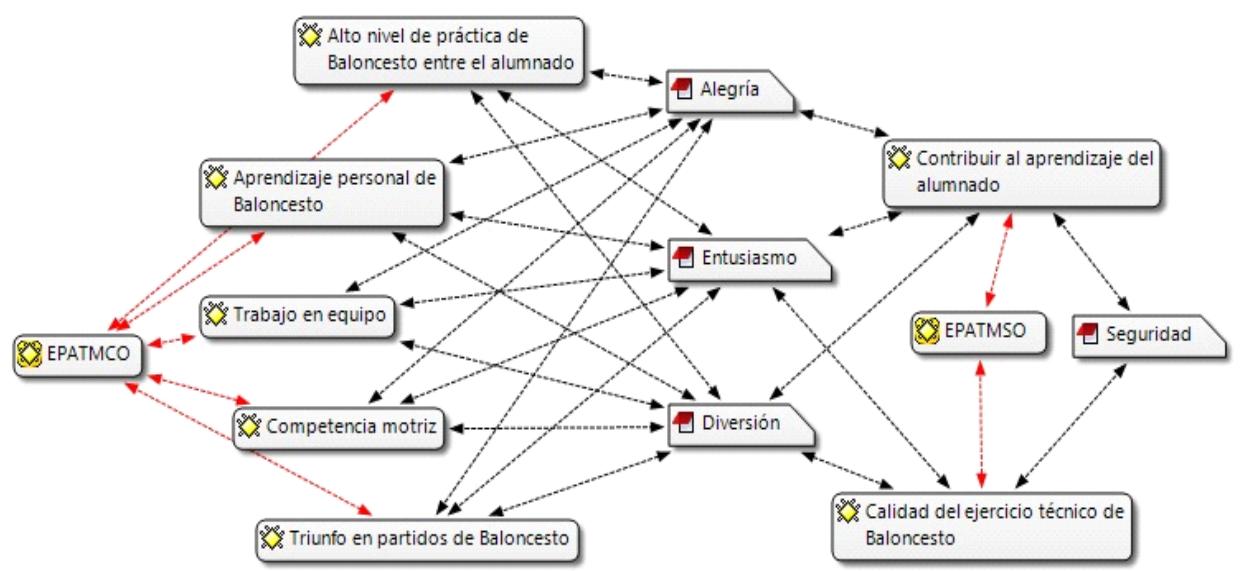

Figura 1. Códigos independientes en función del tipo de tarea motriz. Fuente: Elaboración propia. cesto; h) desafío motor en las prácticas baloncesto; i) trabajo en equipo; j) competencia motriz; k) contribuir al aprendizaje del alumnado; 1) triunfo en partidos de baloncesto; $\mathrm{m}$ ) apoyo entre el alumnado en la práctica de baloncesto; $n$ ) calidad del ejercicio técnico de baloncesto; ñ) práctica autónoma de baloncesto; o) alto nivel de práctica de baloncesto entre el alumnado; p) nivel superior de práctica de baloncesto.

Por otra parte, solo se ha identificado un có-

\section{Emociones positivas según el género}

Las familias de códigos EPAGF y EPAGM presentaron 17 códigos en común. $\mathrm{O}$ sea, que alumnas y alumnos atribuyeron sus emociones a los mismos códigos. En concreto a los siguientes aspectos: a) agrado de practicar baloncesto; b) práctica de baloncesto junto a compañeros y amistades; c) positiva auto-percepción de la práctica de baloncesto; d) convicción de poder lograr ejecutar la tarea de baloncesto; e) aprendizaje personal de baloncesto; f) experiencia previa en baloncesto; g) percepción positiva del desempeño colectivo en la práctica de balon-

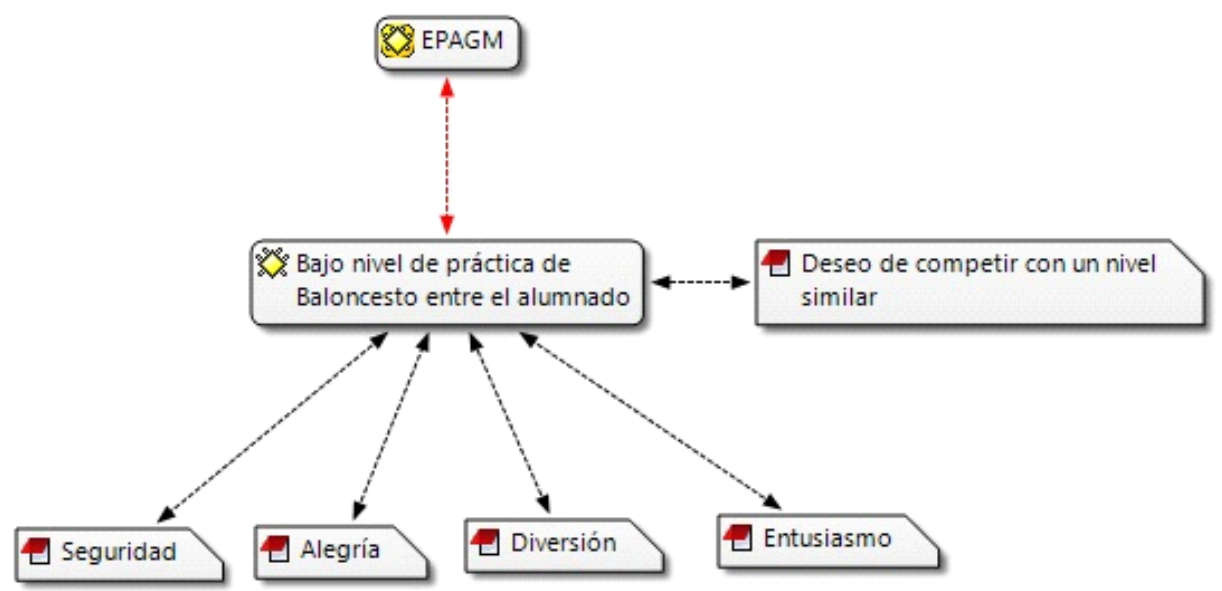

Figura 2. Código independiente en función del género masculino. Fuente: Elaboración propia.

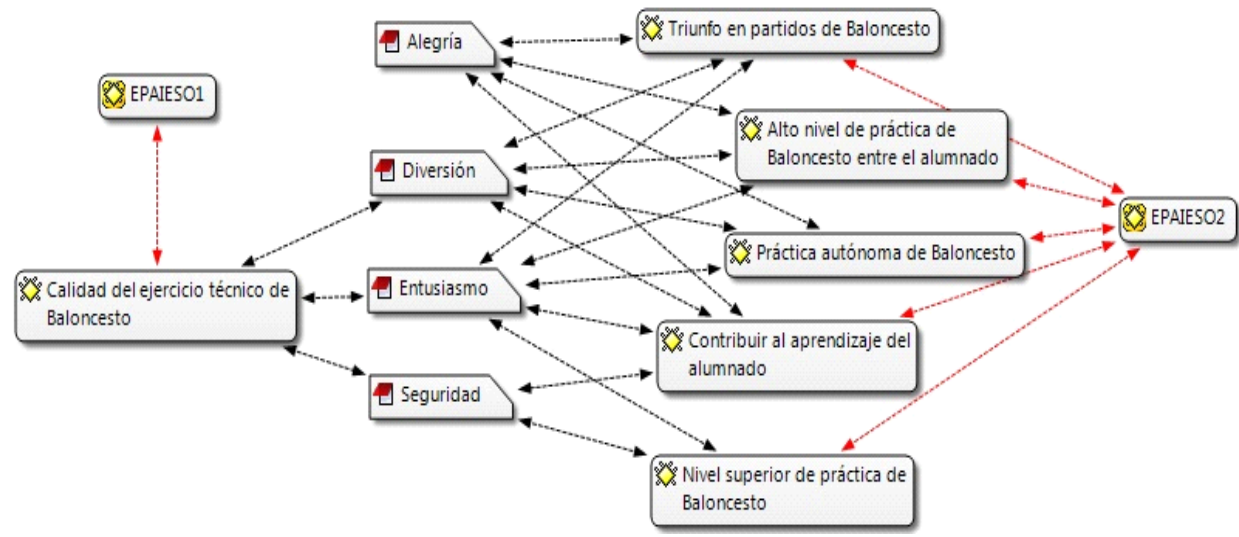

Figura 3. Códigos independientes en función del centro educativo. Fuente: Elaboración propia digo independiente perteneciente a la familia del género masculino. En concreto, dicho código se presenta en la Figura 2. En dicha figura, además del código y las emociones que ha suscitado, se han agregado un deseo que podría explicar este motivo. En concreto, se hace referencia al deseo de competir con otros estudiantes que tengan un mismo bajo nivel de baloncesto, ya que, de esta forma, mejoran las posibilidades para el alumnado de lograr un triunfo.

\section{Emociones positivas según el centro educativo}

Las familias de códigos EPAIESO1 y EPAIESO2 presentaron los siguientes 12 códigos en común: a) agrado de practicar baloncesto; b) práctica de baloncesto junto a compañeros y amistades; c) positiva auto-percepción de la práctica de baloncesto; d) convicción de poder lograr ejecutar la tarea de baloncesto; e) aprendizaje personal de baloncesto; f) experiencia previa en baloncesto; g) percepción positiva del desempeño colectivo en la práctica de baloncesto; h) bajo nivel de práctica de baloncesto entre el alumnado; i) desafío motor en las prácticas baloncesto; j) trabajo en equipo; k) competencia motriz; 1) apoyo entre el alumnado en la práctica de baloncesto.

Por otro lado, dichas familias presentan seis códigos independientes, los cuales se presentan en la Figura 3. 


\section{Discusión}

El agrado por el contenido didáctico de EF desarrollado o, en otras palabras, el gusto hacia el baloncesto, ha sido asociado por parte del alumnado a cuatro emociones positivas para el BS. Igualmente, es la atribución emocional más aludida. Así, este motivo también ha sido reconocido por otros estudios cualitativos, donde estudiantes, en el contexto de EF escolar, señalan sentir emociones positivas para el $\mathrm{BS}$ al disfrutar la práctica de baloncesto y la de otros contenidos didácticos (Dismore \& Bailey, 2011; Leisterer \& Jekauc, 2019; Mujica et al., 2016; Niven et al., 2014). Entre los factores que pueden convertir el baloncesto en un contenido didáctico agradable, encontramos uno revelado en Australia. En concreto, un estudio revela que el alumnado ha destacado explícitamente el reglamento, ya que actuaría como prevención para los comportamientos brutales o descontrolados, de modo que favorecería un buen contexto de aprendizaje motor (Dismore \& Bailey, 2011). En este sentido, es posible señalar que aquella interpretación del alumnado se muestra coherente con el sentido moral que James Naismith, su creador, otorgó al baloncesto (Mujica, 2019). Cabe mencionar, que en este estudio el alumnado no aportó significados que expliquen su agrado por el baloncesto y, por ende, no asoció su agrado específicamente a su reglamento. No obstante, es posible que su agrado planteado se refiera a la totalidad del baloncesto, es decir, a todos sus componentes, incluido la reglamentación que le da un sentido deportivo particular.

Otro aspecto mencionado por el alumnado, que suscita emociones positivas para el BS, es la socialización durante la práctica de baloncesto en EF. En concreto, el alumnado destaca la posibilidad de dialogar, reírse y jugar con sus pares y/o amistades, lo cual también ha sido identificado en estudios similares de EF (Alcaraz-Muñoz et al., 2017; Leisterer \& Jekauc, 2019; Mitchell et al., 2015). Sin embargo, cabe destacar que las interacciones sociales en la UDB se produjeron en un contexto con presencia de valores morales, como el respeto y la solidaridad. Por ello, se infiere que el estudiantado se emociona positivamente ante una socialización de buena calidad humana. Lo anterior queda bien reflejado en otro de los motivos que suscitaron afecto positivo, específicamente, el apoyo entre pares durante la práctica de baloncesto. En cuanto a la presencia de valores morales en EF, es preciso mencionar que, según Dewey (2004), «la moral es tan amplia como los actos que conciernen a nuestras relaciones con los demás. Y potencialmente esto incluye todos nuestros actos, aunque no se piense en sus efectos sociales en el momento de su realización» (p. 297). Por otro lado, algunos estudios han revelado que una socialización de mala calidad moral, es decir, con ausencia de respeto interpersonal y empatía, suscita emociones negativas para el BS (Amado et al., 2015; Camacho-Miñano, 2014; Lodewyk \& Muir, 2017; Monforte \& Pérez-Samaniego, 2017; Mujica-Johnson \& Jiménez, 2020; Timken et al., 2019).

Por otra parte, el alumnado hizo referencia a la buena percepción, individual y colectiva, de competencia motriz, como un factor que suscitó emociones positivas para el BS. Así, dicho resultado refuerza los mismos hallazgos identificados por otras investigaciones en las clases de EF desarrolladas en diferentes continentes (AlcarazMuñoz et al., 2017; Caballero, Alcaraz, Alonso \& Yuste, 2016; Jang, 2015; Jun, 2018; Leisterer \& Jekauc, 2019; Miralles, Filella \& Lavega, 2017; Mujica et al., 2016). Del mismo modo, diferentes estudios cualitativos en EF han relacionado la percepción de incompetencia motriz con las emociones negativas para el BS (Luis-de Cos, ArribasGalarraga, Luis-de Cos \& Arruza, 2019; Mitchell et al., 2015; Monforte \& Pérez-Samaniego, 2017; Timken et al., 2019). En este sentido, se aprecia que, en el aprendizaje motor de baloncesto, es fundamental que el alumnado pueda lograr una buena competencia percibida, para lo cual se requeriría el apoyo afectivo de los pares y del profesorado. Y, sobre todo, estrategias didácticas por parte del profesorado que permitan al alumnado integrarse a la UDB y progresar. De lo contrario, según Ruiz (2014), la competencia motriz del alumnado podría verse tan debilitada que este llegaría a rechazar la materia de EF. Así, entre los principales factores que deberían ser prevenidos, destaca «el constante fracaso, los feedbacks inapropiados, la falta de reconocimiento social y el aislamiento, las expectativas negativas» (Ruiz, 2014, p. 40).

De acuerdo con el tipo de tarea motriz, una de las principales diferencias cualitativas es que solo en las tareas sin oposición el alumnado asoció sus emociones positivas para el BS a trabajo co-educativo. O sea, a participar en el aprendizaje de sus pares, lo cual también se ha manifestado en otros estudios y, específicamente, lo atribuyen a la ausencia de competición que propiciaría la cooperación (Alonso, Marín, Yuste, Lavega \& Gea, 2019; Miralles et al., 2017). Por otra parte, corroborando el análisis anterior, las emociones positivas para el BS durante las tareas con oposición fueron asociadas al resultado de las competencias de baloncesto. Dicha relación entre las emociones positivas y logar ganar una competencia ha sido reconocida en otros estudios realizados en España (Duran et al., 2015; Miralles et al., 2017; Mujica-Johnson \& Jiménez, 2019). Por ello, Miralles et al. (2017) señalan que sería ideal que durante las sesiones de EF todo el alumnado pueda experimentar el placer que produce conseguir un triunfo en la competencia deportiva.

En función del género del alumnado, se ha identificado una diferencia. En concreto, que parte del alumnado masculino atribuyó sus emociones positivas al bajo nivel de práctica de baloncesto de sus pares, ya que eso le posibilitaría competir en similares condiciones de habilidades técnicas y tácticas. Y, a su vez, tendría mayores oportunidades de conseguir la victoria y de no cosechar una derrota. Este resultado revelaría que una parte de los alumnos le estarían otorgan más importancia al resultado de la competencia que las chicas participantes del estudio. Lo cual coincide con otros estudios desarrollados en EF que exponen la mayor preocupación de los chicos por los resultados de la competencia deportiva (AlcarazMuñoz et al., 2017; Lagardera, Lavega, Sáez de Ocáriz, Serna \& Aires, 2011). Además, la investigación de Gaviria y Castejón (2016) ha planteado que aquella actitud centrada excesivamente en la competencia, principalmente 
encarnada por los chicos, puede derivar en un ambiente hostil para las alumnas. En este sentido, otros estudios cualitativos han revelado que algunas alumnas preferirían tener EF solo con estudiantes de su mismo género, ya que los alumnos se mostrarían excesivamente competitivos e irrespetuosos (Casey, Eime \& Payne, 2009; Lodewyk \& Muir, 2017).

Por último, en función del centro educativo, se ha detectado que la cultura de baloncesto del IESO2 ha generado algunas diferencias en las atribuciones emocionales. Principalmente, que solo en este instituto el alumnado ha asociado sus emociones positivas para el BS a un alto nivel de práctica de baloncesto, a la superioridad de la práctica baloncesto y a la co-educación con los pares. Estos resultados confirman la importancia de la cultura deportiva en la afectividad humana durante los procesos de aprendizaje motor que, por cierto, también ha sido identificada en otros estudios (Azzarito et al., 2006; CamachoMiñano \& Aragón, 2014; Gea-García, Alonso-Roque, Yuste-Lucas \& Garcés, 2016; McCaughtry et al., 2006; Monforte \& Pérez-Samaniego, 2017; Mujica-Johnson \& Jiménez, 2019, 2020). No obstante, como bien exponen los estudios estadounidenses de Azzarito et al. (2006) y McCaughtry et al. (2006), el alumnado que se encuentra más implicado en dicha cultura de baloncesto requiere ser orientado desde la perspectiva moral para que su práctica en EF no se torne problemática por el exceso de individualismo. Por ello, en este estudio se aprecia que parte del alumnado ha encarnado el valor de la solidaridad, ya que han puesto su conocimiento y habilidades de baloncesto al servicio del aprendizaje de sus pares.

\section{Conclusiones y aplicaciones prácticas}

De acuerdo con el objetivo de investigación, referido a interpretar la percepción y atribución del alumnado sobre las emociones positivas para el BS durante la práctica de baloncesto en Educación Física, en función del tipo de tarea motriz, el género y el centro educativo, se concluye que en todas las categorías de análisis existieron coincidencias y diferencias cualitativas en torno al significado emocional.

Según las tareas motrices, el género y el centro educativo, las coincidencias se refieren al agrado de practicar baloncesto en EF, a la práctica de dicho deporte junto a sus pares y/o amistades, el buen desempeño personal en las tareas motrices, la percepción de aprendizajes logrados, las experiencias previas en la práctica de baloncesto y el apoyo entre el alumnado.

Las diferencias en función del tipo de tarea motriz, son que parte del alumnado atribuyó sus emociones positivas para el BS a códigos asociados a la competición, el trabajo en equipo y los logros que presentan las tareas con oposición. Por otro lado, parte el alumnado solo asoció sus emociones positivas para el BS a la calidad de los ejercicios técnicos y las acciones co-educativas a las tareas sin oposición. Este último hallazgo sería bastante relevante para comprender el fenómeno del andamiaje o la colaboración pedagógica entre pares, la cual podría ser potenciada ante la ausencia de oposición y competición.
Las diferencias según el género fueron muy reducidas, ya que solo el género masculino atribuyó sus emociones positivas para el Bs al contexto de competición en baloncesto que presenta entre los participantes un nivel similar de habilidades técnico-tácticas.

Sobre las diferencias del centro educativo, se concluye que solo parte del alumnado del IESO2 atribuye sus emociones positivas para el BS a la posibilidad de enseñar a sus pares, a los resultados de las situaciones motrices competitivas, a la autonomía, y al buen nivel de juego en las prácticas de baloncesto. Solo parte del IESO1 asoció sus emociones a las tareas motrices técnicas. En este sentido, es evidente la influencia de la cultura deportiva de cada centro educativo.

A partir de los hallazgos de este estudio, se proponen las siguientes tres aplicaciones prácticas para promover las emociones positivas para el BS en la UDB: a) diseñar la UDB en función de un modelo alternativo de enseñanza del deporte que favorezca las situaciones jugadas de aprendizajes, por ejemplo, los diferentes modelos de enseñanza comprensiva o el modelo de educación deportiva; b) incluir en la UDB un clima de aprendizaje orientado a la tarea, ya que este propicia el refuerzo positivo del profesorado y del alumnado, así como también focaliza la atención en el progreso personal según las propias habilidades; y c) integrar al diseño de la UDB la estrategia adaptativa, la cual permite adaptar diferentes aspectos didácticos a las diferencias individuales del alumnado. Por ejemplo, adaptar las dificultades de las tareas motrices para equilibrar los diferentes niveles de competencia motriz en baloncesto.

\section{Futuras líneas de investigación}

En primer lugar, se considera pertinente indagar específicamente, desde la perspectiva del alumnado, los aspectos cualitativos del baloncesto que suscitan agrado. Y, además, la incidencia que puede tener el reglamento de dicho deporte en la percepción emocional.

En segundo lugar, estudiar con mayor profundidad la percepción de emociones positivas para el BS y los aspectos curriculares, por ejemplo, con especificidad sobre las tareas motrices o los modelos de enseñanza del deporte.

En tercer lugar, se considera pertinente realizar diferentes estudios de casos sobre la experiencia deportiva en baloncesto y las emociones positivas percibidas en la UDB. Igualmente, estos estudios podrían incluir dimensiones como el género, el tipo de experiencia deportiva, el tiempo de especialización en baloncesto, el autoconcepto, la autoestima y/o el historial de conducta ética.

\section{Referencias}

Alcaraz-Muñoz, V., Alonso, J., \& Yuste, J. (2017). Jugar en positivo: Género y emociones en educación física. Apunts. Educación Física y Deportes, 129, 51-63. doi: 10.5672/apunts.2014-0983.es.(2017/3).129.04

Alonso, J. I., Lagardera, F., Lavega, P., \& Etxebeste, J. (2018). Emorregulación y pedagogía de las conductas 
motrices. Acción motriz, 21, 67-76.

Alonso, J., Marín, M., Yuste, J.L., Lavega, P., \& Gea, G. (2019). Conciencia emocional en situaciones motrices cooperativas lúdicas y expresivas en Bachillerato: perspectiva de género. Educatio Siglo XXI, 37(1), 195 212. doi: $10.6018 / \mathrm{j} / 363461$

Amado, D., García, T., Marreiros., López, J., \& del Villar, F. (2015). Analysis of students emotions in agreement with the dance teaching technique used. European Journal of Human Movement, 34, 123-138.

Angulo, J. (2017). Breve introducción a la epistemología. En S. Redon y J. Angulo (Eds.), Investigación cualitativa en educación (pp. 21-40). Buenos Aires: Miño y Dávila.

Azzarito, L., Solmon, M., \& Harrison, L. (2006). «...If I Had a Choice, I Would....». A Feminist Poststructuralist Perspective on Girls in Physical Education. Research Quarterly for Exercise and Sport, 77(2), 222-239. doi: 10.1080/02701367.2006.10599356

Baños, R., Marentes, M., Zamarripa, J., Baena-Extremera, A., Ortiz-Camacho, M., \& Duarte-Felix, H. (2019). Influencia de la satisfacción, aburrimiento e importancia de la educación física en la intención de realizar actividad física extraescolar en adolescentes mexicanos. Cuadernos de Psicología del Deporte, 19(3), 205-215. doi: 10.6018/cpd.358461

Barker, D., Nyberg, G., \& Larsson, H. (2019). Joy, fear and resignation: investigating emotions in physical education using a symbolic interactionist approach. Sport, Education and Society. doi: 10.1080/ 13573322.2019.1672148

Bermúdez, C., \& Sáenz-López, P. (2019). Emociones en Educación Física. Una revisión bibliográfica (20152017). Retos. Nuevas Tendencias en Educación Física, Deportes y Recreación, 36, 597-603. doi: 10.47197/ retos.v36i36.70447

Bisquerra, R. Bisquerra, A., Cabero, M., Filella, G., García, E. López, E... \& Oriol, X. (2016). Educación emocional. Propuesta para educadores y familias. Bilbao: Desclée de Brouwer.

Caballero, M., Alcaraz, V., Alonso, J., \& Yuste, J. (2016). Intensidad emocional en la clase de educación física en función de la victoria: juegos de cooperación-oposición. Revista Electrónica Interuniversitaria de Formación del Profesorado, 19(3), 123-133. doi:10.6018/ reifop.19.3.267291

Cagigal, J. M. (1979). Cultura intelectual y cultura física. Buenos Aires: Kapelusz.

Calderón, E. (2014). Universos emocionales y subjetividad. Nueva Antropología, 27(81), 11-31.

Camacho-Miñano, M., \& Aragón, N. (2014). Ansiedad física social y educación física escolar: las chicas adolescentes en las clases de natación. Apunts. Educación Física y Deportes, 116(2), 87-94. doi: 10.5672/ apunts.2014-0983.es.(2014/2).116.09

Casey, M., Eime, R., Payne, W., \& Harvey, J. (2009). Using a Socioecological Approach to Examine Participation in Sport and Physical Activity Among Rural Adolescent Girls. Qualitative Health Research, 19(7), 881-893. doi:10.1177/1049732309338198
Castejón, F. J. (2010). Deporte como concepto y aplicación. En F. J. Castejón (Ed.), Deporte y Enseñanza Comprensiva (pp. 11-34). Sevilla: Wanceulen.

Denzin, N., \& Lincoln, Y. (Eds.). (2012). El campo de la investigación cualitativa (Vol. I.). Barcelona: Gedisa.

Dewey, J. (2004). Democracia y educación (6 $6^{\mathrm{a}}$ ed.). Madrid: Morata.

Dismore, H., \& Bailey, R. (2011). Fun and enjoyment in physical education: young people's attitudes. Research Papers in Education, 26(4), 499-516. doi: 10.1080/02671522.2010.484866

Duran, C., Lavega, P., Salas, C., Cristofol, T., Tamarit, M., e Invernó, J. (2015). Educación física emocional en adolescentes. Identificación de variables predictivas de la vivencia. Cultura, Ciencia y Deporte, 10(28), 5-18. doi: $10.12800 /$ ccd.v10i28.511

Flick, U. (2015). El diseño de investigación cualitativa. Madrid: Morata.

Fusch, P., Fusch, G., \& Ness, L. (2018). Denzin's Paradigm Shift: Revisiting Triangulation in Qualitative. Journal of Social Change, 10(1), 19-32. doi: 10.5590/ josc.2018.10.1.02

García-González, L., Aibar,A., Sevil, J.,Almolda, F., \& Julián, J. (2015). Soporte de autonomía en Educación Física: evidencias para mejorar el proceso de enseñanza. Cultura, Ciencia y Deporte, 29, 103-111. doi: 10.12800/ ccd.v10i29.547

Gaviria, D., \& Castejón, F. (2016). Desarrollo de valores y actitudes a través de la clase de educación física. Movimento, 22(1), 251-262. doi: 10.22456/19828918.53455

Gea-García, G., Alonso-Roque, J., Yuste-Lucas, J., \& Garcés, E. (2016). Los juegos deportivos y su influencia en la gestión emocional en universitarios. Cuadernos de Psicología del Deporte, 16(3), 101-112.

Gil-Madrona, P., Pascual-Francés, L., Jordá-Espi, A., Mujica-Johnson, F., \& Fernández-Revelles, A. (2020). Afectividad e interacción motriz de los juegos motores populares en la escuela. Apunts. Educación Física y Deportes, 139, 42-48. doi: 10.5672/apunts.20140983.es.(2020/1).139.06

González-Campos, G., Rodríguez-López, M., \& CastañedaVázquez, C. (2018). Evaluación de un programa motivacional de establecimiento de objetivos para la optimización del rendimiento en deportistas de alto nivel: una aproximación cualitativa. Cuadernos De Psicología Del Deporte, 18(3), 12-25.

Hayashi, P., Abib, G., \& Hoppen, N. (2019). Validity in Qualitative Research: A Processual Approach. The Qualitative Report, 24(1), 98-112.

Jang, B. (2015). The Relationships among Middle School Students Approach-Avoidance Temperaments, Emotion, and Engagement in Physical Education Classes. Korean Society of Sport Psychology, 26(3), 95-107.

Jun, M. (2018). The relationship between perceived learning environment and engagement in Physical Education: The multiple mediation effects of multidimensional competence, achievement goals, and emotions. Korean Journal of Teacher Education, 
34(1), 143-163. doi: 10.14333/kjte.2018.34.1.143

Lagardera, F., Lavega, P., Sáez de Ocáriz, U., Serna, J., \& Aires, P. (octubre, 2011). Emociones y género en la práctica de situaciones motrices cooperativas. XIV Seminario Internacional y II Latinoamericano de Praxiología Motriz: Educación Física y contextos críticos. La Plata, Argentina.

Leisterer, S., \& Jekauc, D. (2019). Students' emotional experience in Physical Education. A qualitative study for new theoretical insights. Sports, 7, 1-15. doi: $10.3390 /$ sports7010010

Lodewyk, K., \& Muir, A. (2017). High school females' Emotions, self-efficacy, and attributions during soccer and fitness testing in Physical Education. Physical Educator, 74(2), 269-295. doi: 10.18666/TPE-2017-V74I2-7136

Luis-de Cos, G., Arribas-Galarraga, S., Luis-de Cos, I., \& Arruza, J. (2019). Competencia motriz, compromiso y ansiedad de las chicas en Educación Física. Retos. Nuevas Tendencias en Educación Física, Deportes y Recreación, 36, 231-238.

McCaughtry, N., Barnard, S., Martin, J., Shen, B., \& Hodges, P. (2006). Teachers' perspectives on the challenges of teaching physical education in urban schools. Research Quarterly for Exercise and Sport, 77(4), 486-497. doi: 10.1080/02701367.2006.10599383

Mejía, J. (2011). Problemas centrales del análisis de datos cualitativos. Revista Latinoamericana de Metodología de la Investigación Social, 1(1), 47-60.

Ministerio de Educación, Cultura y Deporte. (2015). Real Decreto 1105/2014, de 26 de Diciembre, por el que se establece el currículo básico de la Educación Secundaria Obligatoria y del Bachillerato. Madrid: Boletín Oficial del Estado.

Miralles, R., Filella, G., \& Lavega, P. (2017). Educación Física emocional a través del juego en educación primaria. Ayudando a los maestros a tomar decisiones. Retos. Nuevas Tendencias en Educación Física, Deporte y Recreación, 31, 88-93.

Mitchell, F., Gray, S., \& Inchley, J. (2015). »This choice thing really works...» Changes in experiences and engagement of adolescent girls in physical education classes, during a school-based physical activity programme. Physical Education and Sport Pedagogy, 20(6), 593-611. doi: 10.1080/17408989.2013.837433

Monforte, J., \& Pérez-Samaniego, V. (2017). El miedo en Educación Física: Una historia reconocible. Movimento, 23(1), 85-99. doi: 10.22456/19828918.71272

Moreno, J., Hernández, A., \& González-Cutre, D. (2009). Complementando la teoría de la autodeterminación con las metas sociales: un estudio sobre la diversión en Educación Física. Revista Mexicana de Psicología, 26(2), 213-222.

Mujica, F. (2019). El sentido moral que James Naismith otorgó al Baloncesto: Una fortaleza para su desarrollo en España y en la Educación Física. EmásF. Revista Digital de Educación Física, 56, 92-103.

Mujica, F. (2020). El término Educación Física en la posmodernidad: contribución de algunas perspecti- vas fenomenológicas. Retos. Nuevas Tendencias en Educación Física, Deportes y Recreación, 38, 795801. doi: 10.47197/retos.v38i38.73011

Mujica, F., Aránguiz, H., Orellana, N., \& González, H. (2016). Atribución emocional de escolares de sexto año básico en la asignatura de Educación Física y Salud. Educación Física y Ciencia, 18(2), 1-6.

Mujica-Johnson, F., \& Jiménez, A. C. (2019). Percepción emocional en la asignatura de Baloncesto de estudiantes del Grado en Ciencias del Deporte: Estudio piloto. Cuadernos de Psicología del Deporte, 19(2), 152-166. doi: 10.6018/cpd.347161

Mujica-Johnson, F., \& Jiménez, A. C. (2020). Percepción emocional del alumnado de $3^{\circ}$ de ESO ante las prácticas de la unidad didáctica de baloncesto en Educación Física. Revista Española de Educación Física y Deportes, 429, 47-60.

Mujica, F., Orellana, N., \& Concha, R. (2018). Atribución emocional en el taller de baloncesto escolar de una escuela pública en Chile: análisis de contenido. Cuadernos de Psicología del Deporte, 18(1), 31-42.

Navarro, E., Jiménez, E., Rappoport, S., \& Thoilliez, B. (2017). Fundamentos de la investigación y la innovación educativa. Logroño: Universidad Internacional de La Rioja.

Niven, A., Henretty, J., \& Fawkner, S. (2014). 'It's too crowded': A qualitative study of the physical environment factors that adolescent girls perceive to be important and influential on their PE experience. European Physical Education Review, 20(3), 35-348. doi: 10.1177/1356336X14524863

Ortín, F., Martín, M., Mena, L., Izquierdo, T., \& López, M. (2018). Análisis cualitativo sobre aspectos psicológicos y rendimiento deportivo en Lucha Olímpica. Revista de Psicología Aplicada al Deporte y al Ejercicio Físico, 3(2), 1-19. doi: 10.5093/rpadef2018a12

Pérez-López, R., Morales-Sánchez, V., Anguera, M., \& Hernández-Mendo, A. (2015). Hacia la calidad de servicio emocional en organizaciones deportivas orientadas a la población infantil: un análisis cualitativo. Revista Iberoamericana de Psicología del ejercicio y el deporte, $10(2), 243-250$

Puig, N. (2012). Emociones en el deporte y sociología. Revista Internacional de Ciencias del Deporte, 8(28), 106-108.

Ruiz, L.M. (2014). De qué hablamos cuando hablamos de competencia motriz. Acción Motriz, 12, 37-44.

Salgado-López, J. I. (2014). As emoções como condicionante didático no ensino dos deportes sociomotores de colaboração-oposição: estudo dos problemas afetivos ligados ao contacto en rugby, andebol e voleibol. (Tesis Doctoral no publicada). Universidade Da Coruña, La Coruña.

Timken, G., McNamee, J., \& Sarah, C. (2019). 'It doesn't seem like PE and I love it': Adolescent girl's views of a health club physical education approach. European Physical Education Review, 25(1), 1-16. doi: 10.1177/ $1356336 \times 17706382$ 